\title{
Improving Motor Coordination in HRI with Bio-Inspired Controllers
}

\author{
Melanie Jouaiti \\ melanie.jouaiti@loria.fr \\ Université de Lorraine, CNRS, Loria \\ Nancy
}

\begin{abstract}
Gestural communication is an important aspect of HRI in social, assistance and rehabilitation robotics. Indeed, social synchrony is a key component of interpersonal interactions which affects the interaction at a behavioral level, as well as at a social level. It is therefore paramount for the robot to be able to adapt to its interaction partner, at the risk of experiencing an awkward interaction. Bio-inspired controllers endowed with plasticity mechanisms can be employed in order to make these interactions as natural and enjoyable as possible. Integrating adaptive properties can lead to the emergence of motor coordination and hence to social synchrony. A non-negligible aspect of the work consists in studying humans in HRI to understand human behavior better and design better interactions. On the long term, this could be quite useful for improved robot-assisted motor therapy.
\end{abstract}

\section{CCS CONCEPTS}

- Human-centered computing; • Computing methodologies $\rightarrow$ Bio-inspired approaches; - Computer systems organization $\rightarrow$ Robotic control; Robotics;

\section{KEYWORDS}

Motor coordination; human-robot interaction; bio-inspired controller

\section{ACM Reference Format:}

Melanie Jouaiti. 2020. Improving Motor Coordination in HRI with BioInspired Controllers. In Companion of the 2020 ACM/IEEE International Conference on Human-Robot Interaction (HRI '20 Companion), March 2326, 2020, Cambridge, United Kingdom. ACM, New York, NY, USA, 3 pages. https://doi.org/10.1145/3371382.3377439

\section{INTRODUCTION}

In rhythmic social interactions, humans experience two physical phenomena which can also be observed in oscillators: the magnet effect entrains both systems until they are coupled and synchronized; the maintenance effect is the struggle of each system to preserve its own intrinsic frequency [9]. These mechanisms could play a fundamental role in interpersonal interactions [6].

Permission to make digital or hard copies of part or all of this work for personal or classroom use is granted without fee provided that copies are not made or distributed for profit or commercial advantage and that copies bear this notice and the full citation on the first page. Copyrights for third-party components of this work must be honored. For all other uses, contact the owner/author(s)

HRI '20 Companion, March 23-26, 2020, Cambridge, United Kingdom

(C) 2020 Copyright held by the owner/author(s)

ACM ISBN 978-1-4503-7057-8/20/03.

https://doi.org/10.1145/3371382.3377439
Social synchrony is at the root of interpersonal interactions $[8,11,21]$ and thus humans expect robots to adapt to them and demonstrate appropriate responses. Robot controllers with adaptive capabilities are therefore necessary for social acceptance. My work is two-fold: first, improve motor coordination in robots using a bio-inspired controller, and second, use this controller for effective motor rehabilitation with autistic children.

\section{RELATED WORKS}

Synchrony is an important aspect of interpersonal interactions which has been evidenced by numerous studies. [17] observed that synchrony has to be bi-directional and may fail if the robot is not adaptive. [19] showed that subjects attributed a greater likability to a partner waving in phase with them. Moreover, in the case of inter-limb coordination, neural synchrony has been observed between cortical areas of the brain [29] but also between two distinct brains [25, 28]. Neuroscientists assume that emotional and social interactions involve a coupling between individuals which is achieved thanks to neural structures with similar properties as those implicated in the neural control of movements.

The consensus that robots should be adaptive leads to the emergence of several methods. Motor coordination in robots can be achieved using Dynamic Movement Primitives [13], such as in [22] where a robotic arm was able to synchronize with an external signal and perform coordinated drum beating with a changing frequency. [2] employed a reservoir of oscillators to reproduce rhythmic arm movements with the NAO robot. While the oscillator can be slightly entrained during the interaction, the oscillators do not retain the frequency, going back to their original properties right afterwards. [5] combined a rhythmic pattern generator (Hopf [12]) and a discrete pattern generator (VITE [3]) to achieve motor coordination.

\section{ACHIEVING MOTOR COORDINATION USING CENTRAL PATTERN GENERATORS (CPGS)}

CPGs are biological neural structures found in the central nervous system of vertebrates or in some ganglia of invertebrates. CPGs are the source of neural activity that controls rhythmic and stereotyped behaviors, such as locomotion, swimming, flying, breathing, swallowing. They can generate a pattern without an input and adapt to an external feedback. Mesoscopic CPGs are usually based on a pair of half-center neurons [10], controlling the extensor and flexor muscles. Half-center oscillators are composed of two neurons which would not have the ability to oscillate individually but their coupling leads to an oscillatory behavior.

The oscillator neurons (See Fig. 1) employed here are based on the Rowat-Selverston neuron which is able to generate both 


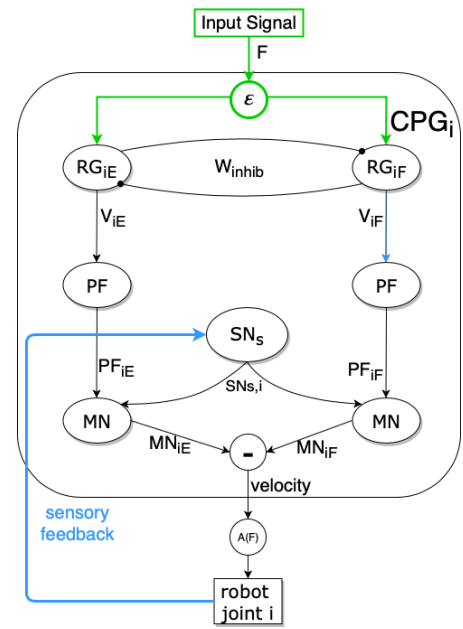

Figure 1: CPG architecture based on the half-center model from [24] for joint $i$. RG: rhythmic generator cells (RowatSelverston here) for the extensor and flexor; PF, MN, SN: layers of interneurons (Pattern Formation, Motoneurons, Sensory Neurons); $\epsilon$ : synaptic weight; W: mutual inhibition. See [14] for a detailed explanation

rhythmic and discrete patterns. In the rhythmic mode, the CPG can adapt its intrinsic frequency, amplitude and synaptic weight using hebbian plasticity mechanisms [14]. This CPG also has a discrete mode where it behaves like a PID controller and it can adapt its parameters to match the input amplitude [15]. The CPG controller confers several advantages: no robot model is necessary; it can take in any kind of input (position, velocity, torque...) and it can achieve coordination through multimodal information (visual, contact...).

In previous work, we compared CPG control with a direct geometric control for human imitation. In discrete mode, the CPG smooths out the signal and is thus less sensitive to noise than the direct geometric control. In rhythmic mode, the CPG adapts its parameters and is able to anticipate the signal. The direct geometric approach endlessly tries to catch up with the signal and never matches the full amplitude.

The adaptation achieved by the CPG is similar to synchronization in humans: the CPG has an adaptation period, synchronization can fail if the input frequency is too different from its own, the CPG still retains its own characteristics while adapting, the CPG can resist synchronization depending on parameters.

The CPG controller is usually coupled with a vision system $[1,4,20]$ to obtain the $3 \mathrm{D}$ pose estimation of the interaction partner, which is then used as an input for the controller (angular position or speed). However, the input can also be the force applied to the robot joints in a handshaking interaction or for collaborative robotics.

Each degree of freedom of the robot is controlled by one CPG and they can be coupled with each other to achieve a bio-inspired architecture.

\section{EVALUATING MOTOR COORDINATION}

Interpersonal coordination can be evaluated thanks to the Phase locking Value (PLV) which has been introduced by [16] to measure coordination in brain signals. It relies on the assumption that both signals are locked with a constant phase difference but the PLV allows for deviation and evaluates its spread. It ranges from 0 (no coordination) to 1 (perfect coordination).

Engagement is "the process by which two (or more) participants establish, maintain and end their perceived connection during interactions they jointly undertake" [26]. It can also be defined as "emotionally laden attention" [7]. Engagement is computed from EEG data [23]:

$$
\text { engagement }=\frac{S P(\beta)}{S P(\alpha)+S P(\theta)}
$$

With $S P(f)$ the spectral power of the frequency band $f[\theta$ (4 $8 \mathrm{~Hz}), \alpha(8-13 \mathrm{~Hz})$ and $\beta(13-30 \mathrm{~Hz})]$. The higher the score, the higher the engagement.

\section{CONCLUSION AND PERSPECTIVES}

In this work, we incorporated plasticity mechanisms into CPG controllers to make them even more adaptive. Versatile control of a robot can be achieved to imitate both discrete and rhythmic movements and switch seamlessly from one to the other. We can thus achieve synchronization in rhythmic tasks but also motor imitation for discrete movements. The model in itself is also quite flexible and can be extended or modified with new plasticity rules better suited for the purpose.

We performed a user-study to evaluate the impact of adaptive controllers on human engagement and coordination. Most participants enjoyed the interaction much more when the partner was adaptive than when it was not, they were very playful and tested the limits of the controller. This experiment also confirmed that humans expect their interaction partner to be able to adapt and feel the lack of adaptation as awkward. Most subjects also declared that they felt the non-adaptive partners adapted to them, demonstrating that the subjects unconsciously coordinated.

In future work, we will perform several user studies to evaluate human perception with CPG control and geometric control in imitation or interaction with contact.

The CPG controller could also be a valuable method for robotassisted motor therapy, as it is both suited for physical interactions with (exoskeletons) and without contact (motor therapy). Besides, these controllers will be employed in a pilot study aimed at motor rehabilitation for autistic children. Motor rehabilitation in ASD is a paramount problematic since motor deficits are correlated with communication deficits [18]. This issue has, however, been a little neglected by the research community with few impactful studies. Combining a robot (with which autistic children more likely engage) and rhythm therapy (the most effective therapy for motor deficits) appears to be a promising idea. [27] compared rhythm therapy and robot-rhythm therapy with autistic children and they reported that the children grew bored with the robot limitations. Could adaptive controllers, such as CPGs, improve the interaction? 


\section{ACKNOWLEDGMENTS}

I thank all the subjects who willingly participated to my studies, Dominique Martinez for proof-reading this paper and Patrick Hénaff for supervision.

\section{REFERENCES}

[1] [n.d.]. https://github.com/ildoonet/tf-pose-estimation.

[2] Eva Ansermin, Ghiles Mostafaoui, Nils Beaussé, and Philippe Gaussier. 2016 Learning to synchronously imitate gestures using entrainment effect. In International conference on simulation of adaptive behavior. Springer, 219-231.

[3] Daniel Bullock and Stephen Grossberg. 1988. The VITE model: a neural command circuit for generating arm and articulator trajectories. Dynamic patterns in complex systems (1988), 305-326.

[4] Zhe Cao, Gines Hidalgo, Tomas Simon, Shih-En Wei, and Yaser Sheikh. 2018 OpenPose: realtime multi-person 2D pose estimation using Part Affinity Fields. arXiv preprint arXiv:1812.08008 (2018).

[5] Sarah Degallier, Ludovic Righetti, Sebastien Gay, and Auke Ijspeert. 2011. Toward simple control for complex, autonomous robotic applications: combining discrete and rhythmic motor primitives. Autonomous Robots 31, 2-3 (2011), 155-181.

[6] Emilie Delaherche, Mohamed Chetouani, Ammar Mahdhaoui, Catherine SaintGeorges, Sylvie Viaux, and David Cohen. 2012. Interpersonal Synchrony: A Survey of Evaluation Methods across Disciplines. IEEE Transactions on Affective Computing 3, 3 (2012), 349-365.

[7] Jacek P Dmochowski, Paul Sajda, Joao Dias, and Lucas C Parra. 2012. Correlated components of ongoing EEG point to emotionally laden attention-a possible marker of engagement? Frontiers in human neuroscience 6 (2012), 112.

[8] Melissa Ellamil, Josh Berson, and Daniel S Margulies. 2016. Influences on and Measures of Unintentional Group Synchrony. Frontiers in psychology 7 (2016) 1744

[9] Eva M Fernández and Helen Smith Cairns. 2017. The Handbook of Psycholinguistics. John Wiley \&amp; Sons.

[10] Sten Grillner and Peter Wallen. 1985. Central pattern generators for locomotion, with special reference to vertebrates. Annual review of neuroscience 8, 1 (1985), 233-261.

[11] Mathieu Gueugnon, Robin N Salesse, Alexandre Coste, Zhong Zhao, Benoît G Bardy, and Ludovic Marin. 2016. Postural coordination during socio-motor improvisation. Frontiers in psychology 7 (2016), 1168.

[12] Eberhard Hopf. 1942. Abzweigung einer periodischen Lösung von einer stationären Lösung eines Differentialsystems. Ber. Math.-Phys. Kl Sächs. Akad. Wiss. Leipzig 94 (1942), 1-22.

[13] Auke Jan Ijspeert, Jun Nakanishi, and Stefan Schaal. 2002. Learning rhythmic movements by demonstration using nonlinear oscillators. In Proceedings of the ieee/rsj int. conference on intelligent robots and systems (iros2002). 958-963.

[14] Melanie Jouaiti, Lancelot Caron, and Patrick Hénaff. 2018. Hebbian plasticity in CPG controllers facilitates self-synchronization for human-robot handshaking. Frontiers in Neurorobotics 12 (2018), 29.
[15] Melanie Jouaiti and Patrick Henaff. 2018. CPG-based Controllers can Generate Both Discrete and Rhythmic Movements. In 2018 IEEE/RSf International Conference on Intelligent Robots and Systems (IROS).

[16] Jean-Philippe Lachaux, Eugenio Rodriguez, Jacques Martinerie, Francisco J Varela, et al. 1999. Measuring phase synchrony in brain signals. Human brain mapping 8, 4 (1999), 194-208.

[17] Tamara Lorenz, Astrid Weiss, and Sandra Hirche. 2016. Synchrony and Reciprocity: Key Mechanisms for Social Companion Robots in Therapy and Care. International fournal of Social Robotics 8, 1 (2016), 125-143.

[18] Megan MacDonald, Catherine Lord, and Dale A Ulrich. 2014. Motor skills and calibrated autism severity in young children with autism spectrum disorder. Adapted physical activity quarterly 31, 2 (2014), 95-105.

[19] C Neil Macrae, Oonagh K Duffy, Lynden K Miles, and Julie Lawrence. 2008. A case of hand waving: Action synchrony and person perception. Cognition 109, 1 (2008), 152-156.

[20] Julieta Martinez, Rayat Hossain, Javier Romero, and James J Little. 2017. A simple yet effective baseline for $3 \mathrm{~d}$ human pose estimation. In Proceedings of the IEEE International Conference on Computer Vision. 2640-2649.

[21] Alexandra Paxton and Rick Dale. 2017. Interpersonal Movement Synchrony Responds to High-and Low-Level Conversational Constraints. Frontiers in psychology 8 (2017), 1135.

[22] Dimitris Pongas, Aude Billard, and Stefan Schaal. 2005. Rapid synchronization and accurate phase-locking of rhythmic motor primitives. In Intelligent Robots and Systems, 2005.(IROS 2005). 2005 IEEE/RSf International Conference on. IEEE, 2911-2916.

[23] Alan T Pope, Edward H Bogart, and Debbie S Bartolome. 1995. Biocybernetic system evaluates indices of operator engagement in automated task. Biological psychology 40, 1-2 (1995), 187-195.

[24] llya A Rybak, Natalia A Shevtsova, Myriam Lafreniere-Roula, and David A McCrea. 2006. Modelling spinal circuitry involved in locomotor pattern generation: insights from deletions during fictive locomotion. The fournal of physiology 577 , 2 (2006), 617-639.

[25] Richard C Schmidt, Claudia Carello, and Michael T Turvey. 1990. Phase transitions and critical fluctuations in the visual coordination of rhythmic movements between people. Fournal of experimental psychology: human perception and performance 16, 2 (1990), 227.

[26] Candace L Sidner, Christopher Lee, Cory D Kidd, Neal Lesh, and Charles Rich. 2005. Explorations in engagement for humans and robots. Artificial Intelligence $166,1-2$ (2005), 140-164.

[27] Sudha M Srinivasan, Maninderjit Kaur, Isabel K Park, Timothy D Gifford, Kerry L Marsh, and Anjana N Bhat. 2015. The effects of rhythm and robotic interventions on the imitation/praxis, interpersonal synchrony, and motor performance of children with autism spectrum disorder (ASD): a pilot randomized controlled trial. Autism research and treatment 2015 (2015).

[28] Emmanuelle Tognoli, Julien Lagarde, Gonzalo C DeGuzman, and JA Scott Kelso. 2007. The phi complex as a neuromarker of human social coordination. Proceedings of the National Academy of Sciences 104, 19 (2007), 8190-8195.

[29] Francisco Varela, Jean-Philippe Lachaux, Eugenio Rodriguez, and Jacques Martinerie. 2001. The brainweb: phase synchronization and large-scale integration. Nature reviews neuroscience 2, 4 (2001), 229. 